\title{
Caracterização do processo de rigor mortis em músculos de cordeiros e carneiros da raça Santa Inês e maciez da carne*
}

\author{
Characterization of rigor mortis process of muscles lamb and yearling mutton \\ of Santa Ines breed and meat tenderness \\ Izabelle de Oliveira ${ }^{1}$, Teófilo José Pimentel da Silva ${ }^{2}$, Mônica Queiroz de Freitas², \\ Rogério Tortelly² \& Flávia de Oliveira Paulino ${ }^{1}$
}

\begin{abstract}
RESUMO
O desenvolvimento do processo de rigor mortis das carcaças dos animais de açougue influenciam diretamente a qualidade da carne. As características do processo de rigor mortis em carcaça de ovinos durante o processamento industrial para obtenção de carcaças resfriadas só foram observadas em outros países e ainda não estabelecidas nas condições brasileiras. Assim, objetivou-se neste trabalho caracterizar o processo de rigor mortis dos músculos Longissimus dorsi e Triceps brachii de 12 carcaças ovinas durante o resfriamento industrial e a maciez da carne. Foram escolhidos aleatoriamente 12 ovinos machos inteiros da raça Santa Inês, sendo seis cordeiros e seis carneiros, abatidos no Matadouro Frigorífico de Campos - Campos dos Goytacazes, Rio de Janeiro. Após a sangria, analisou-se temperatura, pH, comprimento de sarcômero em diferentes intervalos de tempo (1h, 5h, 8h, 10h, 12h, 15h, 24h) e força de cisalhamento ou maciez (48h) dos cortes filé de lombo e paleta. Paralelamente, foi realizada a correlação entre a análise sensorial e a análise instrumental desses cortes. A temperatura da câmara fria variou de $4,2^{\circ} \mathrm{C}(1 \mathrm{~h})$ a $-0,5^{\circ} \mathrm{C}(24 \mathrm{~h})$ e a temperatura média das carcaças foi de $28,58^{\circ} \mathrm{Ce}$ $0,24^{\circ} \mathrm{C}$, respectivamente. $\mathrm{O} \mathrm{pH}$ médio inicial do músculo $L$. dorsi foi 6,67 e o final 5,61 e no músculo T. brachii foi de 6,73 (1h) e 5,68 (24h). A contração máxima do sarcômero do músculo $L$. dorsi ocorreu na $12^{\mathrm{a}}$ hora $(1,45 \mathrm{~mm})$ após a sangria e do músculo T. brachii, na $15^{\mathrm{a}}$ hora $(1,46 \mathrm{~mm})$. O filé de lombo foi mais macio $(\mathrm{p}<0,01)$ que a paleta e a carne de carneiro foi mais dura que a de cordeiro, comparando ambos os cortes. O painel sensorial confirmou o filé de lombo ser o mais macio. Na correlação da análise instrumental (força de cisalhamento) com a análise sensorial, quando comparadas diferentes faixas etárias e dois tipos de cortes, observou-se uma boa correlação inversa $(\mathrm{r}=-0,77)$.
\end{abstract}

Descritores: carcaça ovina, músculos, rigor mortis, maciez.

\begin{abstract}
The development of rigor mortis process of butcher animal carcasses directly influencing the meat quality. The characteristics of rigor mortis process in ovine carcasses during the industrial chilling to obtain the chilled carcasses only were observed in other countries and even no established in brazilians conditions. Thus, this research was designed to characterize the rigor mortis process of Longissimus dorsi and Triceps brachii muscles of 12 ovine carcasses during the industrial chilling and meat tenderness. Twelve intact male ovines Santa Ines breed were randomly assembled, been six lambs and six yearling muttons, slaughtered at Campos Slaughterhouse - Campos dos Goytacazes, Rio de Janeiro. After exsanguination, were measured temperature, $\mathrm{pH}$ and sarcomere length at different times $(1 \mathrm{~h}, 5 \mathrm{~h}, 8 \mathrm{~h}, 10 \mathrm{~h}, 12 \mathrm{~h}, 15 \mathrm{and} 24 \mathrm{~h})$ and shear force or tenderness (48h) of loin roast and arm roast chops. In parallel was accomplished the sensorial analysis relationships to instrumental values of these chops. The chilling room temperature varied between $4.2^{\circ} \mathrm{C}(1 \mathrm{~h})$ to $-0.5^{\circ} \mathrm{C}(24 \mathrm{~h})$, and the mean temperature of carcasses was $28.58^{\circ} \mathrm{C}$ and $0.24^{\circ} \mathrm{C}$, respectively. The mean initial $\mathrm{pH}$ of Longissimus dorsi was 6.67 and final 5.61 and of Triceps brachii was $6.73(1 \mathrm{~h})$ and $5.68(24 \mathrm{~h})$. The maximum contraction of sarcomere of $L$.dorsi occurred at 12th hour $(1.45 \mu \mathrm{m})$ after exsanguination whereas for the Triceps brachii at 15 th hour $(1.46 \mu \mathrm{m})$. The loin roast was tender $(\mathrm{p}<0.01)$ than arm roast and the meat of yearling mutton was tougher than lamb when compared both chops. The sensory panel confirmed the loin roast be more tender. The correlation of instrumental analysis (shear force) when compared different ages and two types of chops was found a good inversed correlation $(r=-0.77)$.
\end{abstract}

Key words: ovine carcass, muscles, rigor mortis, tenderness.

* Projeto financiado com recursos do CNPq e da CAPES. ${ }^{1}$ Programa de Pós-graduação em Medicina Veterinária da Faculdade de Veterinária da Universidade Federal Fluminense - UFF ( Especialidade: Higiene e Processamento Tecnológico de Produtos de Origem Animal). ${ }^{2}$ Departamento de Tecnologia dos Alimentos da Faculdade de Veterinária - UFF. CORRESPONDÊNCIA: T.J.P. Silva [mtatjps@vm.uff.br ; FAX: +55 212714 4041]. 


\section{INTRODUÇÃO}

Um dos primeiros animais a serem domesticados foi o ovino que é encontrado em diferentes áreas geográficas no mundo [9]. O Santa Inês [cruzamento das raças Bergamácia com a Crioula e Morada Nova] é uma raça nordestina oriunda da Bahia de grande porte e prolífera, bem adaptada aos climas quentes e com grande potencial para carne e pele [5].

A queda do $\mathrm{pH}$ e da temperatura durante o processo de rigor mortis das carcaças influenciam diretamente a qualidade da carne. A velocidade do rigor mortis é controlada, principalmente, pela reserva de glicogênio, $\mathrm{pH}$ e temperatura do músculo $[8,11,15]$. A determinação do tamanho de sarcômero demonstra uma correlação positiva entre sua dimensão e o desenvolvimento do rigor mortis, bem como da maciez da carne [20]. Por outro lado, em animais mais velhos ocorre uma diminuição da solubilidade da proteína colágeno, resultando assim em carnes mais duras $[1,7,12,17]$. A análise instrumental (força de cisalhamento) e a análise sensorial são as metodologias mais utilizadas no controle da maciez das carnes $[2,13]$.

As características do rigor mortis de carcaças de ovinos resfriadas só foram observadas em outros países $[4,14,20]$ e ainda não estabelecidas nas condições brasileiras.

Os objetivos do trabalho foram os seguintes: (1) caracterizar o comportamento do processo de rigor mortis nos músculos Longissimus dorsi e Triceps brachii em carcaças frigorificadas; (2) determinar o valor de $\mathrm{pH}$ e do comprimento de sarcômero dos músculos Longissimus dorsi e Triceps brachii e suas mudanças durante a retirada do calor sensível das carcaças na câmara de resfriamento, logo após a sangria; (3) estudar o efeito da idade sobre a maciez da carne nos cortes desses músculos e (4) correlacionar os valores da análise instrumental (força de cisalhamento) com os da análise sensorial.

\section{MATERIAIS E MÉTODOS}

Foram escolhidos ao acaso 12 ovinos machos, inteiros, da raça Santa Inês, sendo 06 com dentes de leite e peso vivo médio de $38,83 \pm 3,18 \mathrm{Kg}$ (cordeiros) e $06 \mathrm{com}$ quatro dentes definitivos e com 52,97 \pm $5,20 \mathrm{Kg}$ (carneiros). A determinação da idade dos animais foi realizada através do exame da dentição no curral de matança e confirmada na sala de matança do Matadouro Frigorífico de Campos, Campos dos Goytacazes - RJ (SIE 504). Os ovinos foram abatidos após os cuidados ante mortem que neste caso incluía o período de repouso, jejum e dieta hídrica de vinte quatro horas antes do abate.

Os animais foram pesados, abatidos, eviscerados e as carcaças foram pesadas e destinadas à câmara de resfriamento $\left(0,8^{\circ} \mathrm{C}\right.$ a temperatura média do ar e $81,2 \%$ a umidade relativa). As meias carcaças foram conduzidas devidamente identificadas para a câmara frigorífica, na qual foi realizada a tomada de temperatura com um termohigrômetro, nos intervalos de tempo de $1 \mathrm{~h}, 5 \mathrm{~h}, 8 \mathrm{~h}, 10 \mathrm{~h}, 12 \mathrm{~h}, 15 \mathrm{~h}$ e $24 \mathrm{~h}$ após a sangria. Nos mesmos intervalos foi tomada a temperatura das meias carcaças introduzindo-se a haste metálica de um termômetro digital na profundidade de $05 \mathrm{~cm}$ da massa muscular na altura do ísquio. A determinação do $\mathrm{pH}$ foi realizada nos intervalos de tempo citados anteriormente com potenciômetro Handylab 1 - Schott, utilizando uma solução homogeneizada com $10 \mathrm{~g}$ da amostra em $100 \mathrm{~mL}$ de água destilada [18].

De cada carcaça foram colhidas duas amostras, nos mesmos intervalos supracitados, dos músculos Triceps brachii (miolo da paleta) e Longissimus dorsi (filé de lombo) para determinação do comprimento de sarcômero. Com auxílio de pinça e bisturi foram retiradas amostras de aproximadamente $2,5 \mathrm{~cm}$ de comprimento por $1,5 \mathrm{~cm}$ de largura e $0,5 \mathrm{~cm}$ de espessura, previamente fixadas por garras metálicas. As garras tinham o objetivo de manter o músculo em condições próximas em que se encontrava na carcaça, evitando contração ou distensão das fibras após sua retirada.

As amostras foram colhidas e identificadas com o número da carcaça, hora da colheita e nome do músculo. Em seguida foram colocadas em frascos plásticos de boca larga contendo formalina tamponada $10 \%$ $(250 \mathrm{~mL})$. Após a fixação as amostras foram clivadas, desidratadas, clarificadas, incluídas em parafina e seccionadas em um micrômetro (Pika - Seiko) com espessura de cinco micra. Os cortes histológicos foram corados com Hematoxilina Fosfotúngstica de Mallory [3].

As lâminas foram lidas em microscopia óptica (Microscópio Nikon) com luz visível em objetiva de imersão [19]. Este método é baseado na contagem de 10 sarcômeros de seis miofibrilas diferentes, com a 
medição do sarcômero sendo feita por uma ocular milimetrada com uma escala de 10 micrômetros. A média obtida foi multiplicada pelo fator de correção da objetiva de imersão (fator encontrado de $0,8 \mu \mathrm{m}$ ) e o valor expresso em micrômetros $(\mu \mathrm{m})$.

Foram colhidas amostras de aproximadamente $250 \mathrm{~g}$ de filé de lombo e de paleta de cada carcaça após $24 \mathrm{~h}$ do abate. Essas amostras foram devidamente identificadas, embaladas e acondicionadas em caixa de isopor, sendo transportadas até o Laboratório de Tecnologia de Carnes da Faculdade de Veterinária da UFF. Nesse Laboratório, 48 horas após a sangria, essas amostras foram cozidas até a temperatura interna de $70^{\circ} \mathrm{C}$ e resfriadas. Posteriormente, foram retirados sete cilindros de cada amostra de $1,27 \mathrm{~cm}$ de diâmetro e cisalhados ao meio na máquina Warner-Bratzler Meat Shear Force - modelo 3000 ${ }^{1}$, para obtenção dos valores da força de cisalhamento [10]. A análise sensorial foi realizada por uma equipe de seis julgadores selecionados e treinados [6].

Para comparação dos valores obtidos utilizouse a análise de variância (delineamento inteiramente casualizado e fatorial), o teste de Tukey e a tendência de correlação linear de Pearson, empregando o programa SAS [16].

\section{RESULTADOS}

Os valores de temperatura das carcaças ovinas foram de $28,58^{\circ} \mathrm{C}(1 \mathrm{~h}), 0,59^{\circ} \mathrm{C}(12 \mathrm{~h})$ e $0,24^{\circ} \mathrm{C}(24 \mathrm{~h})$; enquanto os valores de temperatura da câmara fria foram de $4,2^{\circ} \mathrm{C}(1 \mathrm{~h}), 0,3^{\circ} \mathrm{C}(12 \mathrm{~h})$ e $-0,5^{\circ} \mathrm{C}$ na $24 \mathrm{~h}$ após a sangria (Tabela 1). A análise estatística não revelou diferença significativa $(p>0,01)$ quanto aos valores de temperatura das carcaças nos intervalos de tempo de 12 e $15 \mathrm{~h}, 12$ e $24 \mathrm{~h}$, e 15 e $24 \mathrm{~h}$. Os valores médios encontrados na determinação de $\mathrm{pH}$ dos músculos Longissimus dorsi e Triceps brachii foram de $6,67 \pm 0,04$ e $6,73 \pm 0,05$ na primeira hora, $5,80 \pm 0,06$ e 5,85 $\pm 0,05$ na $12^{a}$ hora e 5,61 $\pm 0,05$ e 5,68 $\pm 0,03$ na $24^{\mathrm{a}}$ hora, respectivamente. As comparações feitas pelo teste de Tukey revelaram não existir diferença significativa $(\mathrm{p}>0,01)$ entre os valores médios de $\mathrm{pH}$ no músculo Longissimus dorsi e Triceps brachii em todos os intervalos de tempo.

Ao estudar as mudanças estruturais nos músculos durante o processo de rigor mortis encontrouse valores médios do comprimento de sarcômero dos músculos Longissimus dorsi e Triceps brachii de 2,25 $\mu \mathrm{m}$ e 1,80 $\mu \mathrm{m}$, respectivamente, após a primeira hora do abate (Tabela 2).

$\mathrm{Na} 12^{\mathrm{a}}$ hora o músculo Longissimus dorsi apresentou seu encurtamento máximo $(1,45 \mu \mathrm{m})$, enquanto o mesmo ocorreu na $15^{\mathrm{a}}$ hora para o músculo Triceps brachii $(1,46 \mu \mathrm{m})$. Na $24^{\mathrm{a}}$ hora o músculo Longissimus dorsi apresentou maior comprimento de sarcômero $(1,70 \mu \mathrm{m})$ que o Triceps brachii $(1,59 \mu \mathrm{m})$.

Na comparação dos valores médios do comprimento de sarcômero no músculo Longissimus dorsi não houve diferença significativa $(p>0,01)$ entre os intervalos de tempo de 10 e $24 \mathrm{~h}, 10$ e $15 \mathrm{~h}, 10$ e $24 \mathrm{~h}$, e

Tabela 1. Valores médios $(X)$ e desvio-padrão (s) de temperatura $\left({ }^{\circ} \mathrm{C}\right)$ de carcaças ovinas e da câmara frigorífica e pH dos músculos Longissimus dorsi (LD) e Triceps brachii (TB), nos diferentes tempos (h) após a sangria de 12 ovinos durante o resfriamento industrial.

\begin{tabular}{|c|c|c|c|c|c|c|c|c|c|}
\hline \multirow[b]{2}{*}{ Análise } & \multirow[b]{2}{*}{ Amostras } & \multicolumn{8}{|c|}{ Tempo após sangria(h) } \\
\hline & & $\mathrm{n}$ & 1 & 5 & 8 & 10 & 12 & 15 & 24 \\
\hline & Câmara & 7 & 4,2 & 6,5 & 3,6 & 0,9 & 0,3 & 0,0 & $-0,5$ \\
\hline Temperatura $\left({ }^{\circ} \mathrm{C}\right)$ & $\begin{array}{c}\text { Carcaça } \\
(\mathrm{X} \pm \mathrm{s})\end{array}$ & 12 & $\begin{array}{c}28,58^{a} \\
4,52\end{array}$ & $\begin{array}{c}12,13^{b} \\
1,50\end{array}$ & $\begin{array}{r}6,26^{c} \\
1,02\end{array}$ & $\begin{array}{l}3,52^{\mathrm{d}} \\
0,86\end{array}$ & $\begin{array}{c}0,59^{\mathrm{e}} \\
0,27\end{array}$ & $\begin{array}{c}0,34^{e} \\
0,12\end{array}$ & $\begin{array}{r}0,24^{\mathrm{e}} \\
0,11\end{array}$ \\
\hline \multirow{2}{*}{$\mathrm{pH}$} & $\begin{array}{l}\text { LD } \\
(X \pm s)\end{array}$ & 12 & $\begin{array}{c}6,67^{a} \\
0,04\end{array}$ & $\begin{array}{l}6,31^{\mathrm{b}} \\
0,07\end{array}$ & $\begin{array}{l}6,09^{c} \\
0,08\end{array}$ & $\begin{array}{l}5,95^{\mathrm{d}} \\
0,02\end{array}$ & $\begin{array}{c}5,80^{\mathrm{e}} \\
0,06\end{array}$ & $\begin{array}{l}5,71^{f} \\
0,06\end{array}$ & $\begin{array}{l}5,61^{9} \\
0,05\end{array}$ \\
\hline & $\begin{array}{l}\text { TB } \\
(X \pm s)\end{array}$ & 12 & $\begin{array}{c}6,73^{a} \\
0,05\end{array}$ & $\begin{array}{c}6,45^{\mathrm{b}} \\
0,11\end{array}$ & $\begin{array}{c}6,20^{\circ} \\
0,11\end{array}$ & $\begin{array}{c}6,01^{\mathrm{d}} \\
0,08\end{array}$ & $\begin{array}{c}5,85^{e} \\
0,05\end{array}$ & $\begin{array}{l}5,76^{e} \\
\text { f0,03 }\end{array}$ & $\begin{array}{l}5,68^{f} \\
0,03\end{array}$ \\
\hline
\end{tabular}

a, b, c, d, e, f, g Médias na mesma linha seguidas de diferentes letras diferem significativamente $(p<0,01)$. 
Tabela 2. Valores médios (X) e desvio padrão (s) de comprimento de sarcômero dos músculos Longissimus dorsi e Triceps brachii nos diferentes tempos (h), após a sangria de 12 ovinos durante o resfriamento industrial.

\begin{tabular}{ccccccccc}
\hline & & \multicolumn{7}{c}{ Comprimento de Sarcômero $(\boldsymbol{\mu m})(\mathbf{X} \pm \mathbf{s}) /$ Tempo $(\mathbf{h})$} \\
\cline { 3 - 9 } Amostra & $\mathrm{n}$ & 1 & 5 & 8 & 10 & 12 & 15 & 24 \\
\hline Longissimus & 12 & $2,25^{\mathrm{aA}}$ & $2,01^{\mathrm{bA}}$ & $1,80^{\mathrm{cA}}$ & $1,60^{\mathrm{dA}}$ & $1,45^{\mathrm{eA}}$ & $1,54^{\mathrm{deA}}$ & $1,70^{\mathrm{cdA}}$ \\
dorsi & & 0,12 & 0,11 & 0,09 & 0,07 & 0,06 & 0,08 & 0,05 \\
Triceps brachii & 12 & $1,80^{\mathrm{aA}}$ & $1,69^{\mathrm{bA}}$ & $1,62^{\mathrm{bCB}}$ & $1,54^{\mathrm{cdA}}$ & $1,51^{\mathrm{cdA}}$ & $1,46^{\mathrm{dA}}$ & $1,59^{\mathrm{cB}}$ \\
& & 0,10 & 0,07 & 0,07 & 0,06 & 0,06 & 0,06 & 0,05 \\
\hline
\end{tabular}

$a, b, c, d, e$ Médias na mesma linha seguidas de diferentes letras diferem significativamente $(p<0,01)$.

A, B Médias na mesma coluna seguidas de mesmas letras não diferem significativamente $(p>0,01)$.

\begin{tabular}{|c|c|c|c|c|c|}
\hline \multirow[b]{3}{*}{ Amostra } & \multirow[b]{3}{*}{$\mathbf{n}$} & \multicolumn{4}{|c|}{ Valores da força de cisalhamento $(\mathrm{Kg})$} \\
\hline & & \multicolumn{2}{|c|}{ Cordeiro } & \multicolumn{2}{|c|}{ Carneiro } \\
\hline & & $x$ & s & $x$ & $\mathrm{~s}$ \\
\hline Filé de lombo & 42 & $2,73^{\mathrm{aA}}$ & 0,14 & $3,55^{\mathrm{bA}}$ & 0,13 \\
\hline Paleta & 42 & $3,77^{\mathrm{aB}}$ & 0,10 & $4,28^{\mathrm{bB}}$ & 0,19 \\
\hline
\end{tabular}

12 e 15h. Em relação ao músculo Triceps brachii não houve diferença significativa nos intervalos de tempo de 5 e 8 h, 8 e $10 \mathrm{~h}, 8$ e $24 \mathrm{~h}, 10$ e $12 \mathrm{~h}, 10$ e $15 \mathrm{~h}, 10$ e $24 \mathrm{~h}, 12 \mathrm{e} 15 \mathrm{~h}$, e 12 . As fotomicrografias do músculo Longissimus dorsi mostram a variação do tamanho de sarcômero durante o desenvolvimento do processo de rigor mortis de $2,25 \mu \mathrm{m}(1 \mathrm{~h})$, de $1,45 \mu \mathrm{m}$ (12h) e de $1,70 \mu \mathrm{m}(24 \mathrm{~h})$ após a sangria (Figura 1).

Os valores médios de força de cisalhamento (FC) para o corte de filé de lombo e para paleta são vistos na Tabela 3. A comparação das médias de força de cisalhamento pelo teste de Tukey demonstrou diferença significativa $(\mathrm{p}<0,01)$ entre os cortes comerciais e idade. O filé de lombo (Longissimus dorsi) dos cordeiros foi mais macio $(\mathrm{p}<0,01)$ do que dos carneiros $(2,73 \mathrm{Kg} \times 3,55 \mathrm{Kg})$ bem como a paleta ou Triceps brachii $(3,77 \mathrm{Kg}$ x $4,28 \mathrm{Kg})$. Quanto ao tipo de corte, o filé de lombo foi mais macio que a paleta, tanto nos cordeiros como nos carneiros.
Os valores da força de cisalhamento encontrados neste estudo apontaram o filé de lombo do cordeiro como a carne mais macia $(2,73 \mathrm{Kg})$ e a paleta do carneiro o corte de maior dureza $(4,28 \mathrm{Kg})$. Valores intermediários foram encontrados para o lombo de carneiro $(3,55 \mathrm{Kg})$ e para a paleta de cordeiro $(3,77 \mathrm{Kg})$.

A correlação linear entre a análise instrumental (força de cisalhamento) e a análise sensorial foi significativa $(\mathrm{p}<0,01)$. A correlação negativa $(r=-0,77)$ demonstra que valores menores da análise instrumental se correlacionam com valores maiores da análise sensorial. A Figura 2 mostra a correlação entre as análises instrumental e sensorial, tendo como base dois cortes (filé de lombo x paleta) e duas idades (cordeiros $\mathrm{x}$ carneiros).

\section{DISCUSSÃO}

O presente estudo encontrou um $\mathrm{pH}$ de 6,09 ( $8^{\mathrm{a}}$ hora post mortem) semelhante ao encontrado por ou- 


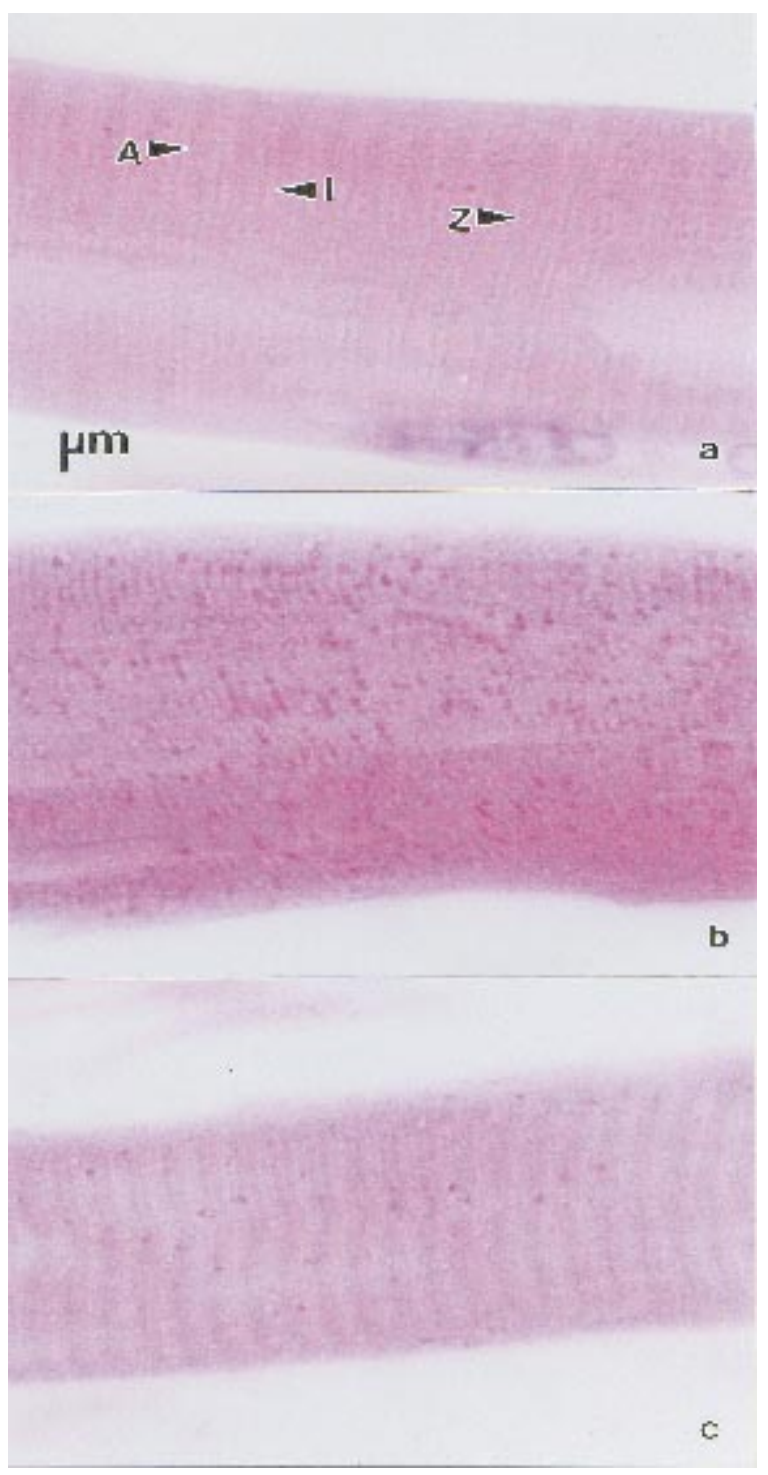

Figura 1. Fotomicrografias do músculo Longissimus dorsi durante o resfriamento industrial, mostrando as bandas A, I eZ (aumento de 1000 vezes), fixado com formalina tamponada neutra e corado com Hematoxilina Fosfotúngstica de Mallory, com diferentes tamanhos de sarcômeros: a) na $1^{\mathrm{a}}$ hora após sangria b) na $12^{a}$ hora e c) na $24^{a}$ hora.

tros autores [8], que estudaram o efeito de três temperaturas de resfriamento $\left(0,16\right.$ e $\left.23^{\circ} \mathrm{C}\right)$ e do $\mathrm{pH}$ na qualidade da carne de cordeiro nas primeiras oito horas post mortem e observaram diferenças significativas no declínio do pH do músculo Longissimus dorsi (6,02, 5,71 e 5,55, respectivamente). Esses resultados retratam que a glicólise anaeróbica ocorre mais rapidamente em temperaturas mais elevadas, em concordância com outro trabalho semelhante [4]. No entanto, essas temperaturas exigem ótimas condições higiênico-sanitárias durante todo o processo de abate e no resfriamento das carcaças, devido ao crescimento microbiano.

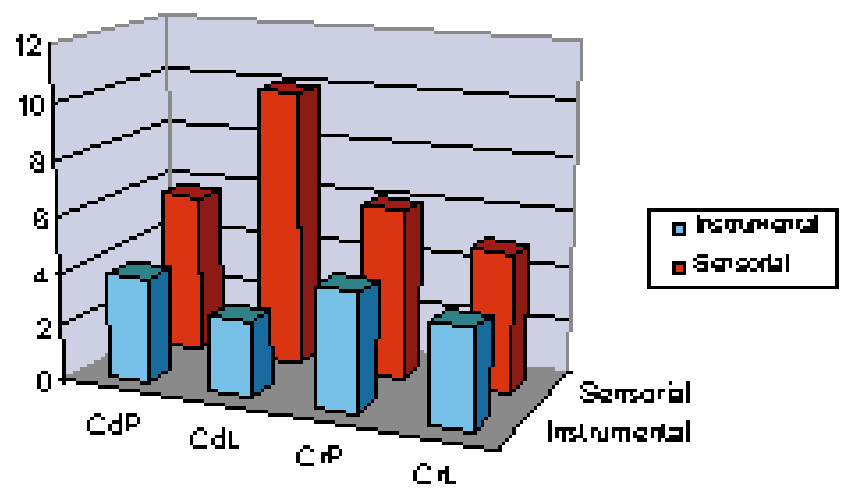

Figura 2. Correlação da análise instrumental (força de cisalhamento) com a análise sensorial, comparando diferentes faixas etárias e dois tipos de corte desses ovinos.

$\mathrm{O}$ valor de $\mathrm{pH}$ final para o músculo Longissimus dorsi foi de 5,61 $\pm 0,05$, enquanto outros autores [15] descreveram valor próximo de $\mathrm{pH}$ final $(5,74 \pm 0,12)$. A proteólise foi estudada [11] durante o período post mortem do músculo Longissimus em carcaças de bovinos, ovinos e suínos e para as carcaças de ovinos foram encontrados valores de temperatura de $39,6^{\circ} \mathrm{C}$ ( 0 hora) e $-0,8^{\circ} \mathrm{C}\left(24^{\mathrm{a}}\right.$ hora) e valores de $\mathrm{pH}$ de 6,89 (0 hora) e 5,65 ( $24^{\mathrm{a}}$ hora). O valor de $\mathrm{pH}$ na $24^{\mathrm{a}}$ hora $(5,65)$ concorda com o obtido neste trabalho $(5,61)$. Por outro lado, o valor da temperatura inicial (0 hora) foi mais elevado $\left(39,6^{\circ} \mathrm{C} \times 28,58^{\circ} \mathrm{C}\right)$. Isso provavelmente se deve ao fato de no presente estudo a tomada da temperatura ter sido realizada na primeira hora após a sangria. Em trabalho semelhante [20] foram estudadas as mudanças prerigor e postrigor no músculo Longissimus dorsi de ovinos; as temperaturas médias observadas foram de $36,4^{\circ} \mathrm{C}$ (0h) e de $1,0^{\circ} \mathrm{C}(24 \mathrm{~h})$ e os valores de $\mathrm{pH}$ inicial de 6,66 e final de 5,81 , em concordância com os resultados obtidos neste estudo.

A velocidade das mudanças bioquímicas que ocorrem no post mortem é influenciada pela temperatura e importante nas características sensoriais da carne. Desse modo, foram encontrados em carcaças de cordeiros resfriadas a $0^{\circ} \mathrm{C}$ por $72 \mathrm{~h}$, tamanho de sarcômero de $1,68 \mu \mathrm{m}$ no músculo Longissimus dorsi, $1,68 \mu \mathrm{m}$ no Biceps femoris e $1,75 \mu \mathrm{m}$ no Semimembranosus [4]. O valor encontrado para o músculo Longissimus dorsi $\left(0^{\circ} \mathrm{C}\right)$ está em concordância com o presente estudo $(1,70 \mu \mathrm{m})$. Trabalho se- 
melhante [8] observou um tamanho de sarcômero de $1,79 \mu \mathrm{m}$ a $0^{\circ} \mathrm{C}, 96 \mathrm{~h}$ após o abate de cordeiros. Esses valores discordam dos encontrados nesta pesquisa, provavelmente em virtude da mensuração ter sido realizada com 96h após o abate, quando a carne deu início ao processo de maturação.

O desenvolvimento do processo de rigor mortis dos animais de açougue demonstra diferentes comprimentos de sarcômeros durante a instalação do processo bioquímico. Assim, foram estudadas as mudanças que ocorreram antes e após o rigor mortis e sua influência na maciez do músculo Longissimus dorsi de ovinos [20]. Esses autores encontraram nesse músculo um comprimento de sarcômero de $2,24 \mu \mathrm{m}$

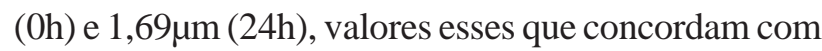
o presente estudo. No entanto, no que diz respeito à contração máxima, os autores acima relatam ter ocorrido na $24^{\mathrm{a}}$ hora enquanto no presente estudo foi na $12^{\mathrm{a}}$ hora (Figura 1). O presente estudo concorda com outros autores [14] de que o processo de rigor mortis dos ovinos é mais rápido que o dos bovinos.

Os valores médios de força de cisalhamento encontrados para o corte de filé de lombo foram de $2,73 \mathrm{~kg}$ (cordeiro) e $3,55 \mathrm{~kg}$ (carneiro). Resultados semelhantes foram relatados por outros autores [12] que encontraram valores de $2,17 \mathrm{Kg}$ a $2,66 \mathrm{Kg}$ no músculo Longissimus de cordeiros Targhee e Sulfock x Targhee; de $2,37 \pm 0,32 \mathrm{~kg}$ no lombo de cordeiros Texel/ Corriedale [15] e valores de $2,3 \mathrm{Kg}$ a $2,8 \mathrm{Kg}$ no mesmo músculo de cordeiros Santa Inês e Bergamácia [5]. Valores de $3,6 \mathrm{Kg}$ a 4,0Kg foram descritos para o lombo de carneiros [2], concordando com a força de cisalhamento observada no presente estudo $(3,77 \mathrm{Kg})$. Por outro lado, valores maiores $(4,69 \mathrm{Kg}$ a $4,97 \mathrm{Kg})$ foram encontrados no músculo vasto lateral de carneiros [1]; e de 4,36Kg para o músculo Longissimus de carneiros [20]. Esses resultados maiores na força de cisalhamento podem ser explicados pelo fato que em animais mais velhos ocorre uma diminuição da solubilidade do colágeno e um aumento na estabilidade dos entrecruzamentos de suas fibras, resultando assim em carnes mais duras [7,17].

Os resultados da análise sensorial confirmam os da análise instrumental, ou seja, o filé de lombo de cordeiro é a carne mais macia. Resultados semelhantes foram descritos para a maciez do músculo peitoral de frango em diferentes tempos após o abate [13], onde também foi observada correlação entre as análises sensorial e instrumental.

\section{CONCLUSÕES}

- A queda da temperatura das carcaças ovinas durante o resfriamento industrial ocorreu dentro dos padrões estabelecidos;

- O declínio do $\mathrm{pH}$ acompanhou o desenvolvimento do processo de rigor mortis em ambos os músculos estudados até a $24^{\mathrm{a}}$ hora após a sangria;

- Durante o processo de rigor mortis a contração máxima do músculo Longissimus dorsi ocorreu na $12^{\mathrm{a}}$ hora e na $15^{\mathrm{a}}$ hora para o músculo Triceps brachii;

- O declínio de pH de ambos os músculos apresentou uma alta correlação inversa entre o tempo após a sangria e uma média correlação inversa entre o comprimento do sarcômero e tempo após a sangria;

- A carne de carneiro foi mais dura do que a dos cordeiros e o filé de lombo (Longissimus dorsi) foi mais macio do que a paleta (Triceps brachii), demonstrando que a idade e tipo de músculo influenciam na maciez da carne;

- Na comparação da análise instrumental versus análise sensorial observou-se uma correlação linear significativa.

\section{Nota Informativa}

${ }^{1}$ G-R Manufacturing Company, 1317 Collins Lane, Manhattan, Kansas, USA. 


\section{REFERENNCIAS}

1 Aalhus J.L. \& Price M.A. 1990. The effect of a progressive-resistance exercise on growth, development and meat quality of sheep. Canadian Journal of Animal Science.70: 89-95.

2 Babiker S.A., El Khider I.A. \& Shafie, S.A. 1990. Chemical composition and quality attributes of goat meat and lamb. Meat Science. 28:273-277.

3 Behmer O.A., Tolosa E.M.C. \& Neto A.G.F. 1976. Manual de técnicas para histologia normal e patológica. São Paulo: EDART - Editora da USP., 239p.

4 Bowling R.A., Smith G.C., Dutson T.R. \& Carpenter Z.L. 1978. Effects of prerigor conditioning treatments on lamb muscle shortening, pH and ATP. Journal of Food Science. 43: 502-507.

5 Bressan M.C., Prado O.V., Pérez J.R.O., Lemos A.L.S.C. \& Bonagurio S. 2001. Efeito do peso ao abate de cordeiros Santa Inês e Bergamácia sobre as características físico-químicas da carne. Ciência e Tecnologia de Alimentos. 21:293303.

6 Damásio M.H. \& Costell E. 1991. Análisis sensorial descriptivo: Generacíon de descriptores y selección de catadores. Revista Agroquímica e Tecnologia de ALimentos. 31: 2: 165-178.

7 Hill F. 1966. The solubility of intramuscular collagen in meat animal of various ages. Journal of Food Science. 31: 161-166.

8 Johnson M.H., Bidner T.D., McMillin K.W., Dugas S.M. \& Hembry F.G. 1989. The effect of three temperature conditioning treatments and subcutaneous fat removal on lamb quality. Journal of Animal Science. 67: 2309-2315.

9 Kammlade Jr. W.G. \& Kammlade W.G. 1955. Sheep Science. Chicago: J.B.Lippincott Co., 536p.

10 Kerth C.R., Miller M.F. \& Ransey C.B. 1995. Improvement of beef tenderness and quality traits with calcium chloride injection in beef loins 48 hours post mortem. Journal of Food Science. 73: 750-756.

11 Koohmaraie M., Whipple, G. Kretchmar D.H., Crouse J.D. \& Mersmann H.J. 1991. Postmortem proteolysis in longissimus muscle from beef, lamb and pork carcasses. Journal of Animal Science. 69: 617-624.

12 Lloyd W.R., Slyter A.L. \& Costello W.J. 1980. Effect of breed, sex and final weight on feedlot performance, carcass characteristics and meat palatability of lambs. Journal of Animal Science. 51: 316-320.

13 Lyon B.G. \& Lyon C.E. 1997. Sensory descriptive profile relationship to shear values of deboned poultry. Journal of Food Science. 62: 885-888.

14 Marsh B.B. \& Thompson J.F. 1958. Rigor mortis and thaw rigor in lamb. Journal Science Food Agriculture. 9: 417-424.

15 Monteiro E.M., Rübensam J. \& Pires G. 2001. Avaliação de parâmetros de qualidade de carcaça e da carne de ovinos. In: Anais do $1^{\circ}$ Congresso Brasileiro de Ciência e Tecnologia de Carnes (São Paulo, Brasil). pp.98-99.

16 SAS Institute. 1985. SAS User's guide. Cary: SAS Institute Inc., 959p.

17 Shimokomaki M., Elsden D.F. \& Bailey A .J. 1972. Meat tenderness: age related changes in bovine intra muscular collagen. Journal of Food Science. 37: 892-896.

18 Silva T.J.P., Orcutt M.W., Forrest J.C., Bracker C.E. \& Judge M.D. 1993. Effect of heating rate on shortening, ultraestructure and fracture behavior of prerigor beef muscle. Meat Science. 33:1-27.

19 Sloss M.W.B.S. \& Kemp R.L.A.B. 1978. Veterinary clinical parasitology. 5th edn. Ames: Iowa State University Press, $247 \mathrm{p}$.

20 Wheeler T.L. \& Koohmaraie M. 1994. Prerigor and postrigor changes in tenderness of ovine longissimus muscle. Journal of Animal Science. 72: 1232-1238. 